\title{
Modified ventricular puncture combined with urokinase in the treatment of secondary intraventricular cast hemorrhage
}

Jian Xu*, Xia Liu, Ze-yu Wu, Xiao-dong Chen, Peng Lun, Yan Zhao, Bing Chen, Chang-qing Ding, Peng Sun and Yi-he Dou

\begin{abstract}
Background: We introduce a new catheter-based minimally invasive approach via frontal tuber for removing hypertensive intraventricular hemorrhage(IVH), and further compare its treatment efficacy with conventional external ventricular drainage (EVD).

Methods: This study is prospective and randomized. Sixty cases of secondary intraventricular cast hemorrhage patients were randomly divided into two groups of 30 cases: modified ventricular puncture (MVP) group and control group. Preoperative Glasgow coma scale (GCS) and Graeb score were compared between the two groups. The postoperative manifestations of two groups were also analyzed and compared statistically, including evacuation rate of intraventricular hematoma in $24 \mathrm{~h}$, the time with drainage tube, rebleeding, complicated by infection, shunt-dependent hydrocephalus and Glasgow outcome scale at 3 months.
\end{abstract}

Results: 1. There was no significant difference in preoperative GCS score and the Graeb score between two groups $(P>0.05)$. 2. In the MVP group via frontal tuber approach, a substantial removal of intraventricular hematoma was achieved in all cases. The average evacuation rate of intraventricular hematoma reached $80.10 \pm 10.16 \%$, the average time of catheter drainage was $3.17 \pm 0.87$ days, the average GOS was $3.80 \pm 0.92$, no intracranial infection and secondary hemorrhage were observed following surgery in all cases, and shunt-dependent hydrocephalus occurred in 2 cases. In the control group, the hematoma evacuation rate was an average of $21.21 \pm 7.81 \%$, the time of drainage was an average of $7.63 \pm 2.87$ days, the GOS was an average of $3.20 \pm 1.12$, intracranial infection after surgery occurred in 5 cases, secondary hemorrhage was observed in 1 case, and shunt-dependent hydrocephalus occurred in 8 cases. Between the two groups there were significantly statistical difference in the hematoma evacuation rate, drainage duration, infection rate and GOS (all $P<0.05$ ).

Conclusions: The new approach is safe, and can quickly remove the hematoma, and significantly shorten the time with a pipe. It can also reduce the occurrence of infection and hydrocephalus, and improve overall outcome of patients, but cannot reduce mortality.

Keywords: Ventricular hemorrhage, Modified ventricular puncture, External ventricular drainage, Hydrocephalus, Urokinase

\footnotetext{
* Correspondence: xujianqdmc@126.com

Department of Neurosurgery, The Affiliated Hospital of Qingdao University, Qingdao, Shandong 266555, China
} 


\section{Background}

Intraventricular hemorrhage (IVH) is considered to be a risk factor for poor prognosis and is characterized by a relatively high mortality rate and morbidity. Clinicians have long been aware of the occurrence of intraventricular hemorrhage (IVH), which for centuries was considered to be uniformly fatal [1-3]. This belief was based on results from autopsy studies, in which patients with an apoplectic onset of neurologic deterioration-progressing to coma, brainstem dysfunction, and death-were found to have blood diffusely within the ventricular system $[1,2,4]$. With the development of computed tomography (CT) scanning, the diagnosis could be made in live patients, and it was realized that individuals with IVH could survive $[1,4,5]$. IVH occurs in 3 to $10 \%$ of all intracerebral hemorrhages $[1,2]$. IVHs can be classified as primary or secondary. Primary IVHs are confined to the ventricular system and immediate parenchymal ependymal lining, originating from intraventricular sources or a lesion in contact with the ventricular wall $[6,7]$. Secondary IVHs originate as an extension of an intraparenchymal or subarachnoid hemorrhage into the ventricular system [6]. About $30 \%$ of IVHs are primary and $70 \%$ are secondary [8]. Our study adopted a modified ventricular puncture external drainage to the treatment of severe secondary intraventricular cast hemorrhage. The goals of this study are aimed to maintain EVD patency, dissolve intraventricular blood clots, reduce ICP and mass effect on the ventricular walls, help clear potentially deleterious blood breakdown products, accelerate patient recovery, decrease the incidence of hydrocephalus, and improve overall outcome.

\section{Methods}

\section{Patients}

From January 2011 to December 2013, 60 patients with secondary intraventricular hemorrhage were treated in our department. All patients were screened and the surgical indication applied to those patients with IVH from parenchymal hemorrhage that caused acute hydrocephalus. Patients with parenchymal hemorrhage not associated with IVH, patients with bleeding tendency, cerebral aneurysm, head trauma, arteriovenous malformation (AVM), vasculitis, hemorrhagic transformation of an ischemic infarct, and tumors were excluded. This study was prospective and randomized. The selected patients were randomly divided into two groups: an EVD group and a modified ventricular puncture (MVP) group. All the patients enrolled have signed the inform consent.

\section{Operation}

In the EVD group, traditional EVDs were performed on patients in the supine position under local anesthesia. Patients with ventricular blood received continuous drainage at $10 \mathrm{~cm} \mathrm{H}_{2} \mathrm{O}$ until there was no further reduction in cerebrospinal fluid (CSF) blood content. The EVD catheter was then sequentially weaned in daily increments of $5 \mathrm{~cm} \mathrm{H}_{2} \mathrm{O}$ and removed. Intraventricular injection of an anticoagulant (urokinase) was given after the procedure.

In the modified ventricular puncture (MVP) group, the surgical procedure was performed with the patient in the supine position. A 2-cm incision was made in the frontal wrinkle scalp ipsilateral to IVH. A burr hole $(0.8 \mathrm{~cm}$ in diameter) was drilled using the navigator system guide. A transcortical transventricular puncture was made with a catheter along the parallel to ventricle direction. When the ventricle was reached, the stylet was removed. Then the catheter continued to enter the depth up to $10 \mathrm{~cm}$, permitting the simultaneous removal of intraventricular hematomas. The catheter was left in the ventricle and urokinase was given after this procedure. The dosage of urokinase is 100,000 units per day, once a day. When all procedures were complete, an EVD was left in the ventricle for $3 \pm 2$ days until there was no further reduction in CSF blood content after which it was removed.

\section{Clinical evaluation}

The clinical evaluation data included the Glasgow Coma Scale (GCS), age, ICH volume, length of the EVD catheter stay, severity of IVH, complicated by infection, shunt-dependent hydrocephalus and Glasgow outcome scale at 3 months. The volume of ICH was calculated using the simplified equation $1 / 2$ ABC. Severity of IVH was graded according to the amount of blood in each ventricle using the Graeb score [4] of 0 to 12 , which is the sum of the score in each ventricle; 4 is the maximum in each lateral ventricle, and 2 is the maximum in the third and fourth ventricles.

\section{Statistical analysis}

All statistical analyses were performed using the SPSS 13.0 Statistics program. A probability value of less than 0.05 was considered statistically significant. Normally distributed data are expressed as the means \pm the standard deviations and were compared using the unpaired $t$ test.

\section{Results}

From January 2011 to December 2013, 60 patients with parenchymal hemorrhage and IVH were enrolled and treated in our department. All the patients had hypertensive hemorrhaging. These patients were randomly divided into an EVD group and an MVP group. The clinical features of each group are shown in Table 1. No significant differences in GCS assessment on admission were found between the two groups. There were no significant differences in ICH volume or Graeb score between the MVP group and the EVD group. Patients in 
Table 1 Comparison of Clinical Data for the two groups $(n=30, x \pm s)$

\begin{tabular}{lllllllll}
\hline & Number & GCS & Evacuation rate $\%$ & Time of drainage & GOS & Infection & Hydrocephalus & Death \\
\hline MVP & 30 & $9.50 \pm 3.57$ & $80.10 \pm 10.16$ & $3.17 \pm 0.87$ & $3.8 \pm 0.92$ & $0 / 30$ & $2 / 30$ & $0 / 30$ \\
EVD & 30 & $9.93 \pm 3.40$ & $21.21 \pm 7.81$ & $7.63 \pm 2.87$ & $3.2 \pm 1.12$ & $5 / 30 / 2015$ & $8 / 30 / 2015$ & $2 / 30$ \\
$P$ value & & $P>0.05$ & $P<0.01$ & $P<0.05$ & $P<0.05$ & $P<0.05$ & $P<0.05$ & $P>0.05$ \\
\hline
\end{tabular}

the MVP group stayed with a catheter for a shorter time $(P<0.05)$ compared with EVD group (Table 1$)$.

In the MVP group via frontal tuber approach, a substantial removal of intraventricular hematoma was achieved in all cases, the average evacuation rate of intraventricular hematoma reached $80.10 \pm 10.16 \%$, the average time of catheter drainage was $3.17 \pm 0.87$ days, the average GOS was $3.80 \pm 0.92$, and no intracranial infection and secondary hemorrhage were observed following surgery in all cases. Shunt-dependent hydrocephalus occurred in 2 cases. In the control group, the hematoma evacuation rate was an average of $21.21 \pm$ $7.81 \%$, the time of drainage was an average of $7.63 \pm$ 2.87 days, and the GOS was an average of $3.20 \pm 1.12$, intracranial infection after surgery occurred in 5 cases, secondary hemorrhage was observed in 1 case, and shunt-dependent hydrocephalus occurred in 8 cases. Between two groups there were significantly statistical difference in the hematoma evacuation rate, drainage duration, infection rate and GOS (all $P<0.05$ ).

Comparisons of preoperative and postoperative $\mathrm{CT}$ scan are shown in the following three Figs. 1, 2 and 3.

\section{Discussion}

Most patients with IVH continue to fare very poorly. It was once believed that the poor prognosis was because of elevated intracranial pressure because of impaired CSF circulation-i.e., the occurrence of obstructive hydrocephalus. In addition to this mechanism, it is now known that the blood clot itself exerts a mass effect on the ventricular walls, and may also cause damage by means of an inflammatory reaction. CT scans may give the false impression that intraventricular blood has resolved, when in actuality it has only become isodense to the CSF normally contained within the ventricles.

Traditionally, treatment of IVH has entailed the placement of one or more external ventricular drains (EVDs) for drainage of blood and CSF from the ventricular system and normalization of intracranial pressure. However, this approach alone is often not sufficiently effective in improving the poor prognosis for severe IVH $[9,10]$. Patency of the EVD may be difficult to maintain. In an effort to keep the EVD open, and to expedite the resolution of the ventricular blood, clinicians have administered fibrinolytic agents directly into the ventricular system. Removal and/or dissolution of the intraventricular hematoma would be expected to decrease intracranial pressure, increase cerebral perfusion, and minimize the chance of IVH-induced hydrocephalus. In selected cases, surgical evacuation of intraparenchymal and intraventricular hematoma has been employed, either through craniotomy or an endoscopic approach.

While the prognosis for IVH is not as dismal as once believed, most patients continue to fare poorly, and improved treatments need to be developed. The morbidity and mortality of patients with IVH depend on: 1) the underlying pathology, 2) the location of the intracranial hematoma (in secondary IVH), and 3) the extent of the intraventricular bleeding $[3,6,7]$. Survival of primary IVH is common, but accompanied by considerable morbidity. Patients with secondary IVH fare more poorly than those with primary IVH $[2,7]$. When an intracerebral hematoma extends into a portion of the ventricular system, the mortality has been reported to be 32 to

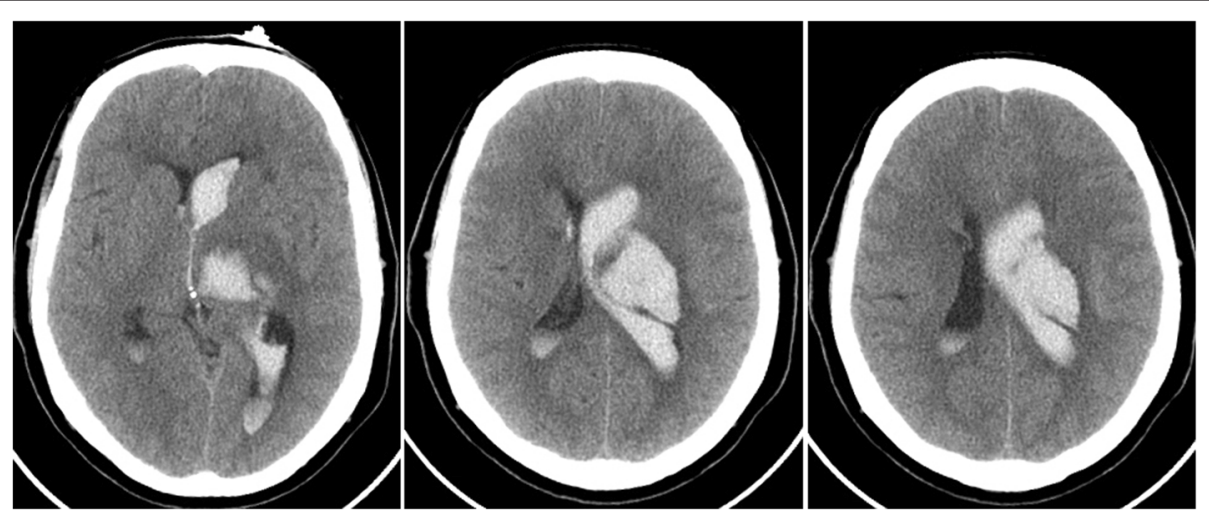

Fig. 1 Preoperative CT scan showed the hemorrhage prior to treatment involved the left thalamus and ruptured into the ipsilateral ventricle 


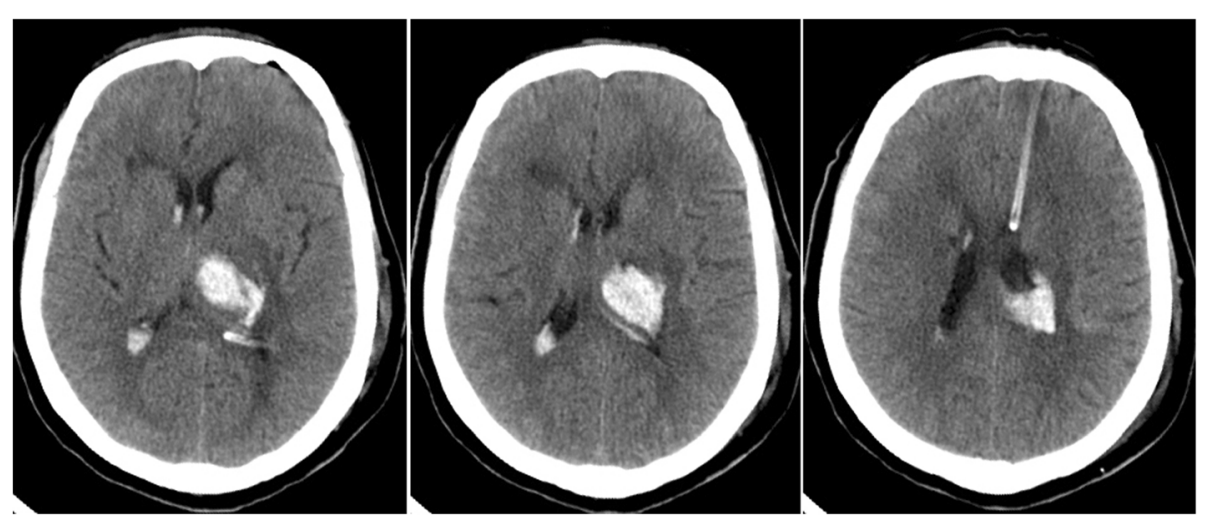

Fig. 2 Repeat CT scan obtained in $24 \mathrm{~h}$ following surgery demonstrated the intraventricular hematoma was almost completely removed with only a small residual clot in posterior horn; The postoperative CT scan depicted the location of the drainage catheter in $24 \mathrm{~h}$ after surgery

$44 \%$. When hemorrhage extends into all four ventricles, the mortality has been 60 to $91 \%[4,7,11,12]$. With respect to the location of the hematoma causing the $\mathrm{IVH}$, patients with hematomas located in the thalamus fare the worst [2].

Among the prognostic factors associated with negative outcome in patients with IVH, the volume of intraventricular blood is certainly one of the most relevant [13]. Therefore, efficient and rapid removal of intraventricular blood is the primary goal in the management of IVH, because it will reduce ventricular dilation and a allow re-equilibration of the CSF circulation. IVH severity influences the occurrence of acute hydrocephalus and the initial level of consciousness, which is significantly associated with prognosis. Priority treatment of IVH should be given to those ICH patients with IVH with Graeb score of 6 or more [13]. Animal studies have demonstrated IVH causes inflammatory response in epidermal and subepidermal tissue layers, as well as inflammation and fibrosis of the arachnoidal villi surface [14]. Therefore, fast removal of ventricular and subarachnoid blood may prevent prolonged irritation of the Pacchioni granulations and ongoing inflammatory response caused by the blood and its breakdown products. This may result in faster recovery of the granulation and avoid persistent hydrocephalus. In our series, the modified ventricular puncture (MVP) group had higher Graeb scores, but the VP shunt rate is lower than in the EVD group. This may have occurred because our procedure can remove the intraventricular blood faster, reduce ventricular dilatation, and reequilibrate the CSF circulation, resulting in the avoidance of persistent hydrocephalus. The main differences between MVP and control method are the puncture point and puncture direction. The MVP's puncture point:eyebrow up $2.5 \mathrm{~cm}$, midline next to $1.5 \mathrm{~cm}$. Puncture direction:parallel to the baseline direction. This point lies between anterial frontal venous and middle frontal venous. This is just the lack of blood vessels. So it is safe.

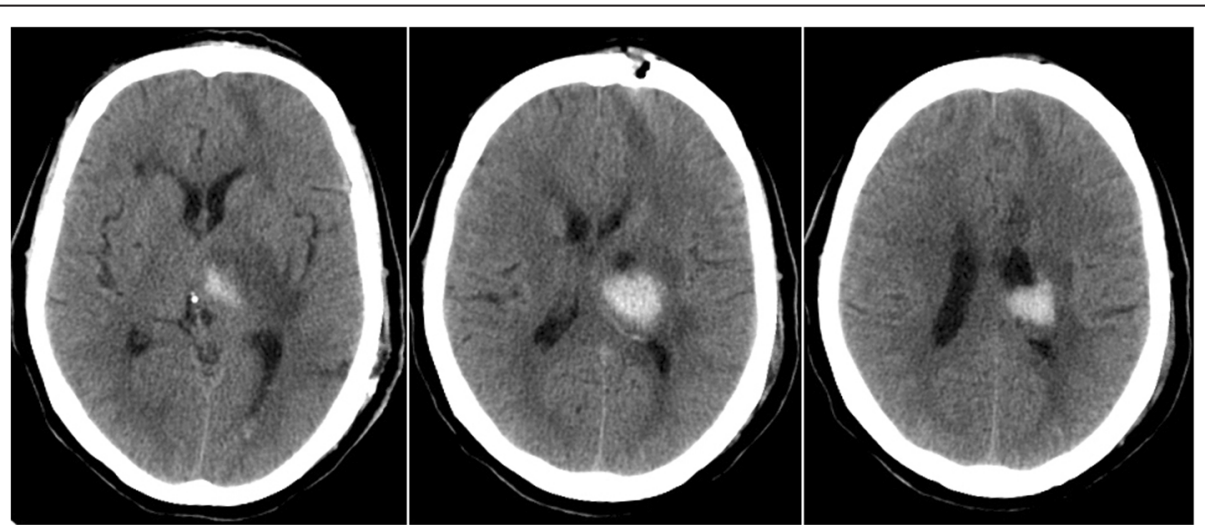

Fig. 3 Follow-up CT scan displayed intraventricular hematoma disappeared completely in 7 day after surgery 
Through this direction, the drainage tube can enter the hematoma along the long axis of the lateral ventricle, and we can suck out the maximum hematoma.

Compared with the traditional EVD, there are many advantages of MVP method. Such as, it's simple and easy to operate, and is conducive to the popularization. It can be carried out in the setting of small local hospitals. It can quickly clear the hematoma and significantly shorten the time with a pipe through MVP method. This would also help reduce the incidence of complications.

\section{Conclusions}

The new approach of modified ventricular puncture external drainage combined with urokinase to the treatment of severe secondary intraventricular cast hemorrhage is safe, quickly removes the hematoma, and significantly shortens the time with a pipe, reduces the occurrence of infection and hydrocephalus, improves overall outcome of patients. Although it cannot reduce mortality, it is warranted for promoting.

\section{Competing interests}

The authors declare that they have no competing interests.

\begin{abstract}
Authors' contributions
$J X, X L$ and ZW carried out the clinical studies, participated in the design of the study and drafted the manuscript. $\mathrm{PL}, \mathrm{XC}$ and $\mathrm{YZ}$ participated in the design of the study and performed the statistical analysis. YD, PS and JX conceived of the study. BC and CD participated in its design and coordination and helped to draft the manuscript. All authors read and approved the final manuscript.
\end{abstract}

\section{Authors' information}

Not applicable.

\section{Acknowledgment}

We acknowledge and thank Zhaojian Li who contributed towards the study by making substantial contributions to conception and design, but who does not meet the criteria for authorship. The sources of funding for all authors are provided by the Qingdao University.

Received: 6 May 2015 Accepted: 17 September 2015

Published online: 18 December 2015

\section{References}

1. Caplan LR. Primary intraventricular hemorrhage. In: Case CS, Caplan LR, editors. Intracerebral hemorrhage. London: Butterworth-Heinemann; 1994. p. 383-401.

2. Darby DG, Donnan GA, Saling MA, Walsh KW, Bladin PF. Primary intraventricular hemorrhage: clinical and neuropsychological findings in a prospective stroke series. Neurology. 1988;38:68-75.

3. Donauer E, Reif J, Mengedoht E-F, Faubert C. Intraventricular hemorrhage caused by aneurysms and angiomas. Acta Neurochir (Wien). 1993;122:23-31.

4. Graeb DA, Robertson WD, Lapointe JS, Nugent RA, Harrison PB. Computed tomographic diagnosis of intraventricular hemorrhage. Etiology and prognosis. Radiology. 1982;143:91-6.

5. LeRoux PD, Haglund MM, Newell DW, Grady MS, Winn HR. Intraventricular hemorrhage in blunt head trauma: an analysis of 43 cases. Neurosurgery. 1992;31:678-85.

6. Findlay $\mathrm{JM}$, Wong $\mathrm{JH}$. Clinical aspects of intraventricular hemorrhage. In: Welch KMA, Caplan LR, Reis D, Siesjo“ BJ, editors. Primer on cerebrovascular diseases. San Diego: Academic; 1997. p. 437-46.

7. Tuhrim S, Horowitz DR, Sacher M, Godbold JH. Volume of ventricular blood is an important determinant of outcome in supratentorial intracerebral hemorrhage. Crit Care Med. 1999;27:617-21.
8. Andrews $\mathrm{CO}$, Engelhard $\mathrm{HH}$. Fibrinolytic therapy in intraventricular hemorrhage: a review of the literature. Annal Pharmacother. 2001;35:1-13.

9. Shapiro SA, Campbell RL, Scully T. Hemorrhagic dilation on the fourth ventricle: an ominous predictor. J Neurosurg. 1994;80:805-9.

10. Slavin KV, Ausman Jl, Charbel FT, Misra M, Munoz LF. Results of the treatment of intraventricular hemorrhage with recombinant tissue plasminogen activator in a series of 10 patients (abstract). J Neurosurg. 1998:88:194A.

11. Jichici D, Frank Jeffrey FI. Thrombolytic therapy in neurointensive care. Crit Care Clin. 1997;13:201-27.

12. Goh KY, Poon WS. Recombinant tissue plasminogen activator for the treatment of spontaneous adult intraventricular hemorrhage. Surg Neurol. 1998;50:526-31.

13. Nishikawa T, Ueba T, Kajiwara M, Miyamatsu N, Yamashita K. A priority treatment of the intraventricular hemorrhage (IVH) should be performed in the patients suffering intracerebral hemorrhage with large IVH. Clin Neurol Neurosurg. 2009;111:450-3.

14. Mayfrank L, Kim Y, Kissler J, Delsing P, Gilsbach JM, Schroder JM, et al. Morphological changes following experimental intraventricular hemorrhage and intraventricular fibrinolytic treatment with recombinant tissue plasminogen activator. Acta Neuropathol. 2000;100:561-7.

\section{Submit your next manuscript to BioMed Central and take full advantage of:}

- Convenient online submission

- Thorough peer review

- No space constraints or color figure charges

- Immediate publication on acceptance

- Inclusion in PubMed, CAS, Scopus and Google Scholar

- Research which is freely available for redistribution 